\title{
Coupled-cluster impurity solvers for dynamical mean-field theory
}

\author{
Tianyu Zhu $\odot,{ }^{1}$ Carlos A. Jiménez-Hoyos, ${ }^{2}$ James McClain, ${ }^{1}$ Timothy C. Berkelbach, ${ }^{3,4, *}$ and Garnet Kin-Lic Chan ${ }^{1, \dagger}$ \\ ${ }^{1}$ Division of Chemistry and Chemical Engineering, California Institute of Technology, Pasadena, California 91125, USA \\ ${ }^{2}$ Department of Chemistry, Wesleyan University, Middletown, Connecticut 06457, USA \\ ${ }^{3}$ Department of Chemistry, Columbia University, New York, New York 10027, USA \\ ${ }^{4}$ Center for Computational Quantum Physics, Flatiron Institute, New York, New York 10010, USA
}

(Received 29 May 2019; revised manuscript received 19 August 2019; published 26 September 2019)

\begin{abstract}
We describe the use of coupled-cluster theory as an impurity solver in dynamical mean-field theory (DMFT) and its cluster extensions. We present numerical results at the level of coupled-cluster theory with single and double excitations (CCSD) for the density of states and self-energies of cluster impurity problems in the oneand two-dimensional Hubbard models. Comparison to exact diagonalization shows that CCSD produces accurate density of states and self-energies at a variety of values of $U / t$ and filling fractions. However, the low cost allows for the use of many bath sites, which we define by a discretization of the hybridization directly on the real frequency axis. We observe convergence of dynamical quantities using approximately 30 bath sites per impurity site, with our largest 4-site cluster DMFT calculation using 120 bath sites. We suggest that coupled-cluster impurity solvers will be attractive in ab initio formulations of dynamical mean-field theory.
\end{abstract}

DOI: 10.1103/PhysRevB.100.115154

\section{INTRODUCTION}

Dynamical mean-field theory (DMFT) $[1,2]$ and its cluster extensions (such as cluster dynamical mean-field theory (CDMFT) $[3,4]$ and the dynamical cluster approximation $[5,6])$ approximate the single-particle Green's function of an interacting quantum lattice Hamiltonian using the self-energy of a self-consistent impurity model. Computing the impurity self-energy and Green's function is thus the main numerical task, and falls to the so-called quantum impurity solver, the focus of this work.

In DMFT impurity models, the impurity sites retain the full interaction, while the rest of the lattice is replaced by a self-consistent hybridization $\boldsymbol{\Delta}(\omega)$. Impurity solvers can be divided into two classes based on how they treat this hybridization. In the first class (diagrammatic), which includes methods such as diagrammatic Monte Carlo [7] and some forms of continuous-time quantum Monte Carlo [8-10], $\boldsymbol{\Delta}(\omega)$ is directly included in the evaluation of the diagrams. In the second class (bath-based), which includes methods such as exact diagonalization (ED) [11,12], configuration interaction (CI) [13], numerical and density matrix renormalization group methods (NRG, DMRG) [14-16], and the variational Gutzwiller ansatz [17], the hybridization is first unfolded into a discrete bath. The impurity and bath sites then together define an impurity Hamiltonian, from which the Green's function and self-energy can be computed from a finite-system simulation. At zero temperature, this usually involves computing the impurity ground-state wave function, and the impurity Green's function as a correlation function. In this work, we will explore an impurity solver based on

\footnotetext{
*tim.berkelbach@gmail.com

$\dagger$ gkc1000@gmail.com
}

coupled-cluster (CC) theory [18], which falls into this second class of solvers.

The bottleneck in all bath-based methods is the number of sites in the impurity Hamiltonian. Even for a small number of impurity sites, one requires several bath sites per impurity site to adequately discretize the hybridization. The exponential complexity of exact diagonalization limits calculations to about 16 sites in total, and thus to only small impurity clusters, typically with no more than 4 impurity sites and 2 bath sites per impurity site. There are two common strategies to ameliorate this bottleneck. The first is to restrict the Hilbert space in which the diagonalization is performed. This was explored by Zgid and Chan with truncated CI [13], which defines a systematic selection of a reduced set of Slater determinants in which to solve the quantum impurity problem. Bravyi [19] provided a formal justification for this truncation, showing that for fixed impurity size, the Green's function can be converged by a linear combination of Gaussian states with a cost that is polynomial in the accuracy. CI solvers significantly extend the size of systems that can be treated by diagonalization methods particularly with respect to the number of bath sites [20-23]. A second strategy is to parametrize the impurity wave function through a nonlinear ansatz. NRG, DMRG, and the variational Gutzwiller approximation adopt this latter approach.

The CC solvers we explore here also correspond to a nonlinear ansatz for the impurity wave function. CC theory has similar strengths to configuration interaction-for example, it can be used at zero temperature as well as finite temperature [24] and with arbitrary interactions and couplings-but it addresses several formal deficiencies of the truncated CI wave function. Most importantly, the CC parametrization is size extensive [25]. This means that (much like tensor networks) one can represent product states of disjoint interacting clusters, with a number of parameters linear in the number of clusters, instead of the exponential number of parameters in exact 
diagonalization. When interactions are not too strong, this makes the CC parametrization exponentially more compact than the CI parametrization for a given accuracy. In ab initio quantum chemistry calculations, CC theory is generally used in place of CI [26], except when there are simultaneously strong interactions and a large number of degenerate sites.

In this work, we study the performance of truncated CC theory as an impurity solver in cluster dynamical mean-field theory calculations. We will use the 1D and 2D Hubbard models, canonical models of correlated materials, as our test systems. We focus on the lowest-order truncation in the CC theory of the Green's function, namely, equation-of-motion CC theory with single and double excitations (EOM-CCSD), a level of theory that is widely implemented and available in quantum chemistry packages. To understand the quality of our low-order CC truncation, we will compare to dynamical quantities obtained from an exact diagonalization solver with small bath discretizations. We will further exploit the low cost of the CC solver to carry out calculations with a large number of bath sites. Overall, we seek to shed light on the regimes in which low-order CC methods are a promising class of impurity solvers for dynamical mean-field theories.

\section{THEORY RECAPITULATION}

\section{A. Cluster dynamical mean-field theory}

Cluster dynamical mean-field theory (CDMFT) has been extensively reviewed [27-29] and we present only a minimal description sufficient for the numerical considerations in our work. We first consider a general translationally invariant lattice Hamiltonian with one- and two-particle interactions,

$$
\hat{H}=\sum_{p q} h_{p q} a_{p}^{\dagger} a_{q}+\frac{1}{2} \sum_{p q r s} V_{p q r s} a_{p}^{\dagger} a_{q}^{\dagger} a_{r} a_{s},
$$

where $p, q, r, s$ label lattice sites (including spin) and $a^{(\dagger)}$ are fermion annihilation (creation) operators. We then consider a computational unit cell $\mathcal{C}$ with $N_{\mathcal{C}}$ cluster sites, that tiles the lattice through a set of translation vectors $\boldsymbol{T}$. Taking $\boldsymbol{k}$ as a corresponding reciprocal space vector taken from an evenly spaced mesh of $N_{k}$ points in the first Brillouin zone of $\mathcal{C}$, the reciprocal space Hamiltonian becomes

$$
\begin{aligned}
\hat{H}= & \sum_{p q \in \mathcal{C}} \sum_{\boldsymbol{k}} \tilde{h}_{p q}(\boldsymbol{k}) \tilde{a}_{p \boldsymbol{k}}^{\dagger} \tilde{a}_{q \boldsymbol{k}} \\
& +\frac{1}{2} \sum_{p q r s \in \mathcal{C}} \sum_{\boldsymbol{k}_{p} \boldsymbol{k}_{q} \boldsymbol{k}_{r}} \tilde{V}_{p q r s}\left(\boldsymbol{k}_{p}, \boldsymbol{k}_{q}, \boldsymbol{k}_{r}\right) \tilde{a}_{p \boldsymbol{k}_{p}}^{\dagger} \tilde{a}_{q \boldsymbol{k}_{q}}^{\dagger} \tilde{a}_{r \boldsymbol{k}_{r}} \tilde{a}_{s \boldsymbol{k}_{p}+\boldsymbol{k}_{q}-\boldsymbol{k}_{r}}
\end{aligned}
$$

with $\tilde{a}_{p k}^{(\dagger)}=\frac{1}{\sqrt{N_{k}}} \sum_{\boldsymbol{T}} a_{p}^{(\dagger)} e^{i k \cdot T}$, where $\tilde{h}$ and $\tilde{V}$ are the matrix elements of $h$ and $V$ in the symmetry-adapted basis. The noninteracting and interacting Green's functions, $\mathbf{g}(\boldsymbol{k}, \omega)$ and $\mathbf{G}(\boldsymbol{k}, \omega)$, are block diagonal in reciprocal space, and are related by the block-diagonal lattice self-energy $\boldsymbol{\Sigma}(\boldsymbol{k}, \omega)$ via the Dyson equation

$$
\mathbf{G}(\boldsymbol{k}, \omega)=[(\omega+\mu) \mathbf{1}-\tilde{\mathbf{h}}(\boldsymbol{k})-\boldsymbol{\Sigma}(\boldsymbol{k}, \omega)]^{-1} .
$$

The cellular Green's function is related to the reciprocal space Green's function by

$$
\mathbf{G}_{\mathcal{C}}(\omega)=N_{k}^{-1} \sum_{\boldsymbol{k}} \mathbf{G}(\boldsymbol{k}, \omega) .
$$

The key quantity to approximate is the lattice self-energy that contains the effects of interactions. In CDMFT, the lattice self-energy is taken to be equal to the self-energy of an impurity with $N_{\mathcal{C}}$ sites, i.e.,

$$
\boldsymbol{\Sigma}(\boldsymbol{k}, \omega)=\boldsymbol{\Sigma}_{\text {imp }}(\omega) .
$$

The impurity model is characterized by a cellular hybridization $\boldsymbol{\Delta}(\omega)$ that describes the delocalization effects between the cell and the lattice. Defining the cellular noninteracting Hamiltonian $\hat{h}_{\mathcal{C}}$ as

$$
\hat{h}_{\mathcal{C}}=\sum_{p q \in \mathcal{C}} h_{p q} a_{p}^{\dagger} a_{q}
$$

the hybridization follows as

$$
\boldsymbol{\Delta}(\omega)=(\omega+\mu) \mathbf{1}-\mathbf{h}_{\mathcal{C}}-\boldsymbol{\Sigma}_{\text {imp }}(\omega)-\mathbf{G}_{\mathcal{C}}^{-1}(\omega) .
$$

The impurity Green's function $\mathbf{G}_{\text {imp }}(\omega)$ is formally defined from the zero-temperature generating functional $W[\mathbf{J}]$

$$
\begin{gathered}
W=\iint \mathcal{D} \mathbf{c} \mathcal{D} \overline{\mathbf{c}} e^{i S(\mathbf{J})}, \\
S=\iint d t d t^{\prime}\left\{\overline { \mathbf { c } } ^ { T } ( t ) \left[\left(i \partial_{t}-\mathbf{h}_{\mathcal{C}}\right) \delta\left(t-t^{\prime}\right)-\Delta\left(t, t^{\prime}\right)\right.\right. \\
\left.\left.+\mathbf{J}\left(t, t^{\prime}\right)\right] \mathbf{c}\left(t^{\prime}\right)\right\}+V[\mathbf{c}, \overline{\mathbf{c}}],
\end{gathered}
$$

where $\mathbf{c}, \overline{\mathbf{c}}$ are vectors of $N_{\mathcal{C}}$ Grassmann variables, $V[\mathbf{c}, \overline{\mathbf{c}}]$ is the interaction contribution to the action $S, \mathbf{G}_{\text {imp }}\left(t, t^{\prime}\right)=$ $\delta W / \delta \mathbf{J}\left(t, t^{\prime}\right)$, and $\mathbf{G}_{\text {imp }}(\omega)=\frac{1}{2 \pi} \int d t \mathbf{G}_{\text {imp }}(0, t) e^{i \omega t}$.

From the impurity Green's function, $\boldsymbol{\Sigma}_{\text {imp }}(\omega)$ follows as

$$
\boldsymbol{\Sigma}_{\text {imp }}(\omega)=\left[(\omega+\mu) \mathbf{1}-\mathbf{h}_{\mathcal{C}}-\boldsymbol{\Delta}(\omega)\right]-\mathbf{G}_{\text {imp }}^{-1}(\omega) .
$$

Using $\boldsymbol{\Sigma}_{\text {imp }}(\omega)$ as the lattice self-energy in (5) leads to a new lattice Green's function and hybridization, and thus a new impurity Green's function. The self-consistency in CDMFT is then achieved when the cellular Green's function and impurity Green's function agree,

$$
\mathbf{G}_{\text {imp }}(\omega)=\mathbf{G}_{\mathcal{C}}(\omega) .
$$

Note that after self-consistency, if the primitive cell of the lattice is smaller than the computational cell, the cellular Green's function may break translational invariance. There are various related formulations [30,31] that restore the translational invariance (including the dynamical cluster approximation $[5,6])$, but we will not use them here.

In the bath-based CDMFT, the hybridization function is represented by a set of fictitious bath sites and couplings. Formally, we consider each element of $\boldsymbol{\Delta}(\omega)$ as the Hilbert transform [32]

$$
\boldsymbol{\Delta}(\omega)=\int d \varepsilon \frac{\mathbf{J}(\varepsilon)}{\omega-\varepsilon}
$$

with the spectral density

$$
\mathbf{J}(\omega)=-\frac{1}{\pi} \operatorname{Im} \Delta(\omega+i \eta) .
$$


A bath parametrization can be considered a discrete representation of the above integral, where $\varepsilon$ and $\mathbf{J}(\varepsilon)$ take on a discrete set of values given by the bath energies and couplings. One common choice is to approximate $\boldsymbol{\Delta}(\omega)$ along the imaginary axis where it is smooth and can be more easily fitted to a bath representation by numerical optimization of a cost function [11]. This is beneficial for exact diagonalization (and related) solvers which can only handle a very small number of bath sites but can lead to a loss of accuracy when analytically continuing to the real axis for spectral computation [13,3335]. Another choice is to approximate $\Delta(\omega)$ along the real axis directly, which is commonly done with NRG and DMRG solvers [16,36-38]. Here we view the Hilbert transform as a quadrature along the real axis,

$$
\boldsymbol{\Delta}(\omega)=\sum_{n=1}^{N_{\omega}} w_{n} \frac{\mathbf{J}\left(\varepsilon_{n}\right)}{\omega-\varepsilon_{n}}
$$

where $w_{n}$ are quadrature weights for $N_{\omega}$ integration grid points. Then, we can define an approximate hybridization of the form

$$
\tilde{\Delta}_{p q}(\omega)=\sum_{n=1}^{N_{\omega}} \sum_{k=1}^{N_{\mathcal{C}}} \frac{V_{p, k}^{(n)} V_{q, k}^{(n)}}{\omega-\varepsilon_{n}}
$$

where $\mathbf{V}^{(n)}=\left[w_{n} \mathbf{J}\left(\varepsilon_{n}\right)\right]^{1 / 2}$. This leads to the discrete impurity Hamiltonian,

$$
\hat{H}_{\mathrm{imp}}=\hat{H}_{\mathcal{C}}+\sum_{n=1}^{N_{\omega}} \sum_{k=1}^{N_{\mathcal{C}}}\left[\varepsilon_{n} a_{n k}^{\dagger} a_{n k}+\sum_{p}\left(V_{p, k}^{(n)} a_{p}^{\dagger} a_{n k}+\text { H.c. }\right)\right] \text {, }
$$

where $\hat{H}_{\mathcal{C}}$ is the cellular Hamiltonian with interactions and the creation and annihilation operators indexed by $n k$ in the last two terms act on the fictitious bath space. From the impurity Hamiltonian, the Green's functions can then be defined as correlation functions. For example, the addition and removal parts of the impurity Green's function $\mathbf{G}_{\mathbf{i m p}}(\omega)$ are given by

$$
\begin{aligned}
& G_{p q}^{+}(\omega)=\left\langle\Psi_{0}\left|a_{p}\left[\omega+\mu-\left(\hat{H}_{\mathrm{imp}}-E\right)+i \eta\right]^{-1} a_{q}^{\dagger}\right| \Psi_{0}\right\rangle, \\
& G_{p q}^{-}(\omega)=\left\langle\Psi_{0}\left|a_{q}^{\dagger}\left[\omega+\mu-\left(E-\hat{H}_{\mathrm{imp}}\right)+i \eta\right]^{-1} a_{p}\right| \Psi_{0}\right\rangle .
\end{aligned}
$$

The goal of the impurity solver in the bath-based representation is thus to approximate the impurity model groundstate wave function $\left|\Psi_{0}\right\rangle$ and the impurity Green's function $\mathbf{G}_{\text {imp }}(\omega)$ via the expressions in (17). We next describe how this is done in $\mathrm{CC}$ theory.

\section{B. Coupled-cluster theory of the ground state and Green's function}

We now describe the basics of $\mathrm{CC}$ theory. Detailed discussions of ground-state CC and the CC Green's function can be found in Refs. [39-44]. The CC ansatz is an exponential parametrization of the wave function,

$$
\left|\Psi_{0}\right\rangle=e^{\hat{T}}\left|\Phi_{0}\right\rangle
$$

where $\left|\Phi_{0}\right\rangle$ is a reference determinant, and $\hat{T}$ is the cluster excitation operator

$$
\begin{aligned}
\hat{T} & =\sum_{i a} t_{i}^{a} a_{a}^{\dagger} a_{i}+\frac{1}{4} \sum_{i j a b} t_{i j}^{a b} a_{a}^{\dagger} a_{b}^{\dagger} a_{i} a_{j}+\cdots \\
& =\hat{T}_{1}+\hat{T}_{2}+\cdots
\end{aligned}
$$

where indices $i, j, \ldots$ and $a, b, \ldots$ label particle (p) and hole (h) orbitals in the reference determinant, and $t_{i}^{a}, t_{i j}^{a b}, \ldots$ are 1p1h, 2p2h, etc., cluster amplitudes. Truncating the CC amplitudes at $1 \mathrm{p} 1 \mathrm{~h}, 2 \mathrm{p} 2 \mathrm{~h}$, etc., gives the coupled-cluster singles (CCS), coupled-cluster singles and doubles (CCSD), etc., approximations. CCS constitutes a mean-field ansatz. Thus we use the CCSD ansatz, the lowest truncation that includes correlations, in this work.

Given a Hamiltonian $\hat{H}$, the reference determinant $\left|\Phi_{0}\right\rangle$ is often chosen to be the mean-field (Hartree-Fock) ground state of $\hat{H}$ (although including $e^{\hat{T}_{1}}$ in the cluster operator renders the approximation somewhat insensitive to the choice of determinant, as an arbitrary determinant satisfies $\left|\Phi^{\prime}\right\rangle=$ $\left.e^{\hat{T}_{1}}\left|\Phi_{0}\right\rangle\right)$. The amplitudes are chosen to satisfy the projected CC equations; for CCSD, these are

$$
\begin{aligned}
& E=\left\langle\Phi_{0}\left|e^{-\hat{T}} \hat{H} e^{\hat{T}}\right| \Phi_{0}\right\rangle, \\
& 0=\left\langle\Phi_{i}^{a}\left|e^{-\hat{T}} \hat{H} e^{\hat{T}}\right| \Phi_{0}\right\rangle, \\
& 0=\left\langle\Phi_{i j}^{a b}\left|e^{-\hat{T}} \hat{H} e^{\hat{T}}\right| \Phi_{0}\right\rangle,
\end{aligned}
$$

where $\left\langle\Phi_{i}^{a}\right|=\left\langle\Phi_{0}\right| a_{i} a_{a}^{\dagger} \ldots$ and $E$ is the (nonvariational) CC approximation to the ground-state energy. The operator $e^{\hat{T}}$ defines a similarity transformation. Thus $\bar{H}=e^{-\hat{T}} \hat{H} e^{\hat{T}}$ is an effective Hamiltonian whose mean-field energy is $E$, or equivalently, the $\mathrm{CC}$ wave function is a product state of similarity transformed quasiparticles, defined by the quasiparticle operators $\bar{a}_{i}^{\dagger}=e^{-\hat{T}} a_{i}^{\dagger} e^{\hat{T}}$.

The advantages of truncated $\mathrm{CC}$ versus truncated $\mathrm{CI}$ derive from the exponentiation of the cluster operator. A CI wave function can be written in similar notation as

$$
\begin{aligned}
\left|\Psi_{0}\right\rangle & =\hat{C}\left|\Phi_{0}\right\rangle, \\
\hat{C} & =1+\sum_{i a} c_{i}^{a} a_{a}^{\dagger} a_{i}+\frac{1}{4} \sum_{i j a b} c_{i j}^{a b} a_{a}^{\dagger} a_{b}^{\dagger} a_{i} a_{j}+\cdots \\
& =1+\hat{C}_{1}+\hat{C}_{2}+\cdots
\end{aligned}
$$

The truncated $\hat{C}$ can be seen as a linearization of a truncated $e^{\hat{T}}$, or equivalently, the terms in $\hat{T}$ are the cumulants of $\hat{C}$, rewriting the latter as products of cluster excitations, e.g., $\hat{C}_{2}=\frac{1}{2} \hat{T}_{1}^{2}+\hat{T}_{2}$. The extensivity of the truncated CC approximation also derives from the exponential structure, since for a system $A B$ consisting of two separated clusters of sites $A$ and $B$, we can write $e^{\hat{T}_{A B}}\left|\Phi_{A B}\right\rangle=e^{\hat{T}_{A}}\left|\Phi_{A}\right\rangle e^{\hat{T}_{B}}\left|\Phi_{B}\right\rangle$.

If the interactions between particle-hole excitations are not too strong, we expect higher-order cluster operators to become small. In this situation, the truncated CC approximation provides a significant improvement over the truncated $\mathrm{CI}$ ansatz. Low-order CC truncations are accurate if the state of interest can be described in terms of low-order products of fluctuations around the chosen reference mean-field state 
$\left|\Phi_{0}\right\rangle$. In this work, we will consider two possible CCSD formulations based on different reference mean-field states corresponding to restricted Hartree-Fock (RHF) and unrestricted Hartree-Fock (UHF). In restricted CCSD (RCCSD), the reference mean-field state is required to be a singlet $(S=0)$ eigenfunction of $\hat{S}^{2}$, and the cluster operators $\hat{T}_{1}, \hat{T}_{2}$ each commute with $\hat{S}^{2}$. In unrestricted CCSD (UCCSD), the reference mean-field state is only required to be an eigenfunction of $\hat{S}_{z}$, and the cluster operators commute with $\hat{S}_{z}$. Consequently, spin symmetry can be broken in UCCSD, for example, to yield an antiferromagnetic state. RCCSD is expected to be most accurate when the interacting state can be described by fluctuations around a paramagnetic state, while UCCSD is more appropriate to describe fluctuations around an (anti)ferromagnetic state.

From the CC ground state, we then compute the Green's function as a correlation function. Using the cluster operator, similarity transformed Hamiltonian, and quasiparticle operators, we can rewrite the exact expressions for the Green's function in (17) in the equation-of-motion coupled-cluster (EOM-CC) form [45-47]:

$$
\begin{aligned}
G_{p q}^{+}(\omega)= & \sum_{m n}\left\langle\Lambda_{0}\left|\bar{a}_{p}\right| \Phi_{m}\right\rangle\left\langle\Phi_{n}\left|\bar{a}_{q}^{\dagger}\right| \Phi_{0}\right\rangle \\
& \times\left\langle\Phi_{m}\left|\left[\omega+\mu-\left(\bar{H}_{\mathrm{imp}}-E\right)+i \eta\right]^{-1}\right| \Phi_{n}\right\rangle, \\
G_{p q}^{-}(\omega)= & \sum_{m n}\left\langle\Lambda_{0}\left|\bar{a}_{q}^{\dagger}\right| \Phi_{m}\right\rangle\left\langle\Phi_{n}\left|\bar{a}_{p}\right| \Phi_{0}\right\rangle \\
& \times\left\langle\Phi_{m}\left|\left[\omega+\mu-\left(E-\bar{H}_{\mathrm{imp}}\right)+i \eta\right]^{-1}\right| \Phi_{n}\right\rangle,
\end{aligned}
$$

where $\left\langle\Lambda_{0}\right|$ is the left eigenstate of $\bar{H}$, and $\sum_{m}\left|\Phi_{m}\right\rangle\left\langle\Phi_{m}\right|=$ 1 , where $\left|\Phi_{m}\right\rangle$ is a determinant. Defining response vectors $\left|R_{p}^{ \pm}(\omega)\right\rangle$,

$$
\begin{aligned}
& \left|R_{q}^{+}(\omega)\right\rangle=\hat{P}^{+}\left[\omega+\mu-\left(\bar{H}_{\mathrm{imp}}-E\right)+i \eta\right]^{-1} \hat{P}^{+} \bar{a}_{q}^{\dagger}\left|\Phi_{0}\right\rangle, \\
& \left|R_{p}^{-}(\omega)\right\rangle=\hat{P}^{-}\left[\omega+\mu-\left(E-\bar{H}_{\mathrm{imp}}\right)+i \eta\right]^{-1} \hat{P}^{-} \bar{a}_{p}\left|\Phi_{0}\right\rangle,
\end{aligned}
$$

where $\hat{P}^{ \pm}$is the projector onto $1 \mathrm{p}, 2 \mathrm{p} 1 \mathrm{~h}, \ldots$ states or $1 \mathrm{~h}, 2 \mathrm{~h} 1 \mathrm{p}$, ... states, allows the CC Green's functions to be efficiently computed as

$$
\begin{aligned}
& G_{p q}^{+}(\omega)=\left\langle\Phi_{0}\left|\bar{a}_{p}\right| R_{q}^{+}(\omega)\right\rangle, \\
& G_{p q}^{-}(\omega)=\left\langle\Phi_{0}\left|\bar{a}_{q}^{\dagger}\right| R_{p}^{-}(\omega)\right\rangle .
\end{aligned}
$$

A truncated EOM-CC approximation to the Green's function contains two truncations, one of the $\hat{T}$ operator that defines the ground state, and another of the resolution of the identity determinants in the Lehmann sum. In this work we will use the EOM-CCSD approximation, where $\hat{T}$ corresponds to the ground-state CCSD truncation, and where $\left|\Phi_{m}\right\rangle,\left|\Phi_{n}\right\rangle$ are restricted to $1 \mathrm{~h}, 2 \mathrm{~h} 1 \mathrm{p}\left(\mathbf{G}^{-}\right)$and $1 \mathrm{p}, 2 \mathrm{p} 1 \mathrm{~h}\left(\mathbf{G}^{+}\right)$ excitations out of the reference determinant. Note that

$$
\begin{aligned}
& \bar{a}_{p}\left|\Phi_{0}\right\rangle=\left(a_{p}+\left[a_{p}, \hat{T}\right]\right)\left|\Phi_{0}\right\rangle, \\
& \bar{a}_{p}^{\dagger}\left|\Phi_{0}\right\rangle=\left(a_{p}^{\dagger}+\left[a_{p}^{\dagger}, \hat{T}\right]\right)\left|\Phi_{0}\right\rangle ;
\end{aligned}
$$

thus the truncation of $\hat{T}$ at the CCSD level implies that $\bar{a}_{p}\left|\Phi_{0}\right\rangle$ and $\bar{a}_{p}^{\dagger}\left|\Phi_{0}\right\rangle$ are expressible in terms of $1 \mathrm{~h}, 2 \mathrm{~h} 1 \mathrm{p}$ and $1 \mathrm{p}, 2 \mathrm{p} 1 \mathrm{~h}$ spaces, respectively.

From the Green's function one can compute all oneparticle expectation values (such as the particle number and the single-particle density matrix) as well as the total energy, from the Migdal formula [48] $E=-\frac{1}{2 \pi} \operatorname{Im} \int_{-\infty}^{\mu} d \omega \operatorname{Tr}(\omega \mathbf{1}+$ h) $\mathbf{G}(\omega)$. However, EOM-CCSD is not a conserving approximation [49]; thus the energy computed using the Migdal formula is different from the ground-state CC energy $E$. Nonetheless, the single-particle density matrix $\gamma_{p q}=$ $(2 \pi i)^{-1} \int_{-\infty}^{\mu}\left[G_{p q}^{-}(\omega)+G_{p q}^{+}(\omega)\right]$ is correctly normalized and equal to its definition as an energy derivative of the CC energy functional. Furthermore, the EOM-CCSD Green's function is not strictly causal. One implication of this is that the impurity self-energy calculated via Eq. (10) may not have an imaginary part that is negative definite, similar to the behavior observed in adaptively truncated CI solvers [23]. A further discussion of this point and a partial solution are described in the Appendix.

The computational cost of ground-state CCSD for a general (e.g., quantum chemistry) two-particle interaction is $O\left(N^{6}\right)$, where $N$ is the number of sites [more specifically, the cost is $O\left(o^{3} v^{3}+o^{2} v^{4}\right)$, where $o$ is the number of electrons and $v=N-o$ is the number of unoccupied states]. There are some special considerations, however, when using $\mathrm{CC}$ to determine the ground state of the CDMFT impurity Hamiltonian. The impurity Hamiltonian only has two-particle interactions on the impurity sites when working in the site basis. However, the CC equations are usually implemented in the mean-field molecular orbital basis, for which the impurity model has two-particle interactions over all orbitals. Although we do not take advantage of it here, in principle, using the locality of the interaction in the site basis can significantly lower the computational cost of CCSD, particularly if we consider the scaling of the cost when increasing the number of bath sites while the number of impurity sites is kept fixed. Another consideration is that the CC theory presented here is a zero-temperature pure state theory, with a fixed particle number. This is in contrast to the grand canonical formulation of (C)DMFT in Sec. II A. This means that it is necessary to search over all particle numbers (and spin sectors) in the ground-state calculations of the impurity to find the quantum numbers of the (lowest energy) ground state, as done with other zero-temperature impurity solvers [13].

The cost of EOM-CCSD (without accounting for locality in the interaction) is $O\left(N^{5}\right)$ per response vector and thus $O\left(N_{\omega} N_{\mathcal{C}} N^{5}\right)$ for all elements of the frequency-dependent impurity Green's function. Computing the response vector can be done either by solving a system of linear equations or by computing $\left|R_{p}^{ \pm}(t)\right\rangle$ in the time domain followed by Fourier transformation (similarly to what is done in td-DMRG solvers $[50,51])$. We have found that a generalized minimum residual (GMRES) solver [52] or a simplified two-parameter generalized conjugate residual method with inner orthogonalization and outer truncation $[\operatorname{GCROT}(m, k)][53]$ works well for the linear equations and converges in $O(10)$ iterations when the response vector at a nearby frequency is used to initialize the solution for the response vector at a new frequency and the matrix diagonal is used as a preconditioner. 
Once the impurity Green's function is computed, it may then be used in all the expressions in Sec. II B. We note that the paramagnetic formulation of CDMFT is incompatible with symmetry breaking that may occur in the UCCSD impurity solver. To study a paramagnetic phase using the UCCSD solver, we spin-average the impurity Green's function, $\mathbf{G}_{\mathrm{imp}}=$ $\sum_{\sigma=\uparrow, \downarrow} G_{\mathrm{imp}}^{\sigma \sigma}$.

\section{Algorithm}

For completeness, we outline the full computational procedure for our CDMFT algorithm at fixed chemical potential using a CC solver. The loop is initialized with the HartreeFock hybridization.

(1) Discretize hybridization via (29).

(2) Solve impurity problem with Hartree-Fock at fixed $\mu$ (electron number can change).

(3) Calculate impurity Green's function with CC via (23) and (24) using GMRES or GCROT $(m, k)$.

(4) Calculate hybridization $\boldsymbol{\Delta}(\omega)$ via (7) and check for convergence. If not converged, update hybridization (using DIIS [54]) and return to 1.

If results are desired at a fixed occupancy, then the occupancy can be calculated via $n=\operatorname{Tr} \boldsymbol{\gamma}_{\text {imp }} / N_{\mathcal{C}}$ using the groundstate CC solution. If the occupancy does not equal the target occupancy, then the chemical potential is updated and the CDMFT loop is repeated.

We have implemented the above algorithm using the HF, CCSD, and EOM-CCSD routines from the PySCF quantum chemistry package [55].

\section{APPLICATIONS TO THE 1D AND 2D HUBBARD MODELS}

The Hubbard model [56] is defined by the lattice Hamiltonian

$$
\hat{H}=-t \sum_{\langle p q\rangle, \sigma} a_{p \sigma}^{\dagger} a_{q \sigma}+U \sum_{p} n_{p \uparrow} n_{p \downarrow},
$$

where $n_{p \sigma}=a_{p \sigma}^{\dagger} a_{p \sigma}$. It is the canonical model for DMFT studies as there are no nonlocal interactions.

\section{A. Implementation in the 1D and 2D Hubbard models}

We will consider 1 and 2 site clusters for the 1D Hubbard model and 1 and 4 site $(2 \times 2)$ clusters for the 2D Hubbard model. The 2 site and 4 site clusters contain additional point group symmetry which allows us to define a symmetryadapted impurity orbital basis, associated with operators

$$
\begin{aligned}
& a_{\Gamma_{+}}^{\dagger}=\frac{1}{\sqrt{2}}\left(a_{1}^{\dagger}+a_{2}^{\dagger}\right), \\
& a_{\Gamma_{-}}^{\dagger}=\frac{1}{\sqrt{2}}\left(a_{1}^{\dagger}-a_{2}^{\dagger}\right),
\end{aligned}
$$

for two sites, and

$$
\begin{aligned}
& a_{\Gamma_{1}}^{\dagger}=\frac{1}{2}\left(a_{1}^{\dagger}+a_{2}^{\dagger}+a_{3}^{\dagger}+a_{4}^{\dagger}\right), \\
& a_{\Gamma_{2}}^{\dagger}=\frac{1}{2}\left(a_{1}^{\dagger}+a_{2}^{\dagger}-a_{3}^{\dagger}+a_{4}^{\dagger}\right),
\end{aligned}
$$

$$
\begin{aligned}
& a_{\Gamma_{3}}^{\dagger}=\frac{1}{2}\left(a_{1}^{\dagger}-a_{2}^{\dagger}-a_{3}^{\dagger}+a_{4}^{\dagger}\right), \\
& a_{\Gamma_{4}}^{\dagger}=\frac{1}{2}\left(a_{1}^{\dagger}-a_{2}^{\dagger}+a_{3}^{\dagger}-a_{4}^{\dagger}\right),
\end{aligned}
$$

for four sites [57,58]. The impurity Green's function, selfenergy, and hybridization are diagonal in the symmetryadapted orbital basis. The diagonal hybridization leads to a simpler form of the bath discretization (15), which can now be written as

$$
\tilde{\Delta}_{\Gamma}(\omega)=\sum_{n=1}^{N_{\omega}} \sum_{k=1}^{N_{\mathcal{C}}} \frac{\left|V_{\Gamma, n k}\right|^{2}}{\omega-\varepsilon_{n}} .
$$

The one-electron couplings in the site basis are $V_{p, n k}=$ $\sum_{\Gamma} U_{p, \Gamma} V_{\Gamma, n k}$, where $\mathbf{U}$ is the frequency-independent matrix of symmetry-adapted eigenvectors associated with the change of basis.

The bath energies and weights used to discretize the hybridization are chosen according to Gauss-Legendre quadrature on the interval $[-7 t+U / 2,+7 t+U / 2]$ for the $1 \mathrm{D}$ Hubbard model and $[-9 t+U / 2,+9 t+U / 2]$ for the $2 \mathrm{D}$ Hubbard model. In almost all calculations, we achieve convergence using a small imaginary broadening $\eta / t=0.1$. In some strong-coupling cases where convergence was especially challenging, we use a slightly large value $\eta / t=0.2$. This latter value results in a weaker hybridization and also facilitates the solution of the $\mathrm{CC}$ linear response equations (23), similarly to previous observations with DMRG solvers [36]. At convergence, quantities on the real frequency axis are plotted with a larger broadening $\eta / t=0.5$, for visual clarity only.

\section{B. 1D Hubbard model}

We first consider the 1D Hubbard model. We present results at $U / t=2$ and $U / t=6$ (weak and strong coupling relative to the single-particle bandwidth of $4 t$ ). We show the impurity density of states (DOS) on the real frequency axis $\rho(\omega)=-\left(\pi N_{\mathcal{C}}\right)^{-1} \operatorname{Tr} \operatorname{Im} \mathbf{G}(\omega)$ and the imaginary part of the impurity self-energy on the imaginary frequency axis $\operatorname{Im} \Sigma_{11}\left(i \omega_{n}\right)$. For visual clarity, the DOS is plotted with a broadening of $\eta / t=0.5$.

We first assess the accuracy of CCSD compared to exact diagonalization. In Fig. 1, we show the DOS and self-energy from single-site DMFT with 9 bath sites and from two-site CDMFT with 4 bath sites per impurity site (8 in total). Both correspond to an impurity problem with 10 sites which is readily accessible with exact diagonalization. At both values of $U / t$, the RCCSD and ED plots are indistinguishable. Note that at small $U / t$, UHF does not break spin symmetry and UCCSD and RCCSD give identical results. At large $U / t$, UHF strongly breaks spin symmetry; however, the mean-field AFM order is reduced by UCCSD such that the final results are almost indistinguishable from those of RCCSD. Therefore, all results for the 1D Hubbard model are presented for RCCSD only.

We next assess convergence of the DOS with respect to the number of bath sites, which requires impurity problem sizes beyond the reach of ED. In Fig. 2, we show the singlesite DMFT DOS and self-energy computed using 9, 19, and 29 bath sites and the RCCSD solver. The largest impurity 

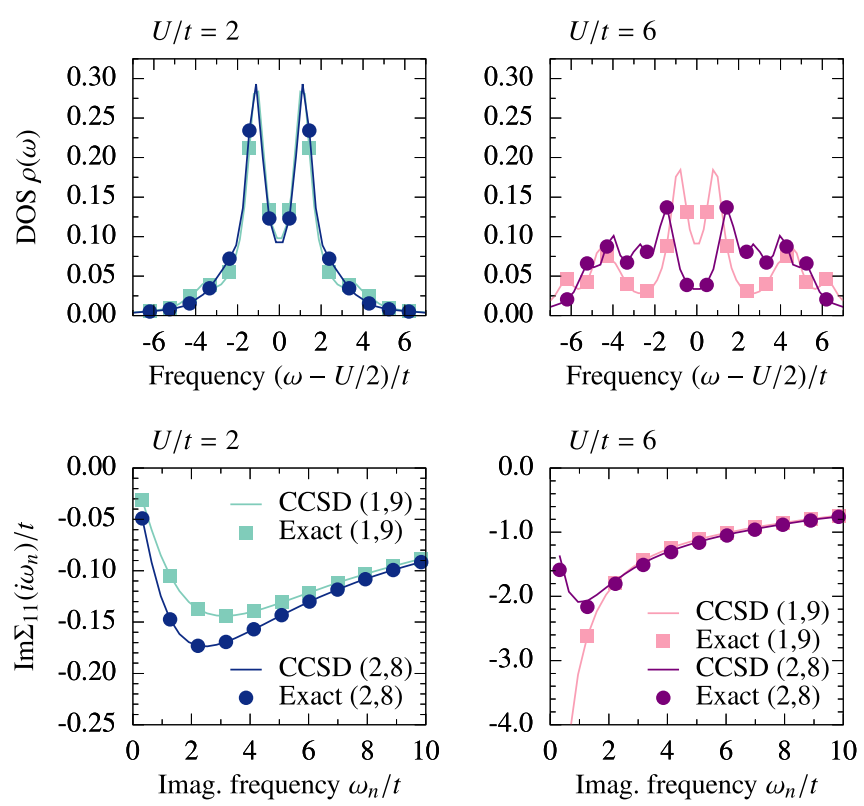

FIG. 1. Single-site DMFT and two-site CDMFT results for the 1D Hubbard model, comparing the use of the CC and exact diagonalization (ED) impurity solvers. Numbers in parentheses indicate the number of impurity sites and the total number of bath sites. Results are shown at half filling with weak interactions $(U / t=2$, left $)$ and strong interactions $(U / t=6$, right). The chemical potential is fixed at $\mu=U / 2$ and the DOS is plotted with a broadening of $\eta=0.5 t$ for clarity.

problem involves 30 particles in 30 orbitals. The plots are qualitatively converged with 19 bath sites and converged to the eye with 29 bath sites. Consistently with previous studies $[20,59,60]$, we find that the single-site DMFT produces a Kondo-like resonance in the DOS at large $U$, as compared with exact Mott insulating behavior at all $U$. We note that this Kondo-like behavior is a result of using a single impurity site in DMFT and is not due to the CCSD approximation.

Based on the converged results for single-site DMFT, we next use two-site CDMFT with at least 30 bath sites per impurity, i.e., 60 bath sites in total. In Fig. 3, we present the DOS as a function of occupancy. At half filling $(n=1, \mu=U / 2)$, we see a clear Mott gap proportional to $U / t$. We access other filling fractions by changing the chemical potential. At small $U / t$, only minor changes are seen in the DOS for moderate changes in the occupancy. At larger $U / t$, there is significant redistribution of the spectral weight toward lower energy, creating a metallic DOS around the chemical potential, which is indicated by a vertical line in the DOS plots. In the bottom panel of Fig. 3, we show the occupancy as a function of the chemical potential for the two values of $U$ studied. The discrete nature of the bath allows access to only a discrete set of occupations, and so these latter data were obtained using larger bath sizes. The appearance of a Mott plateau and suppressed compressibility is clearly seen for $U / t=6$. Due to the discretized bath, there are artificial plateaus in the regions where the Bethe ansatz (BA) varies monotonically, even with 40 bath sites per impurity site. By increasing the bath size to 80 and 60 bath sites per impurity site for $U / t=2$ and 6 ,
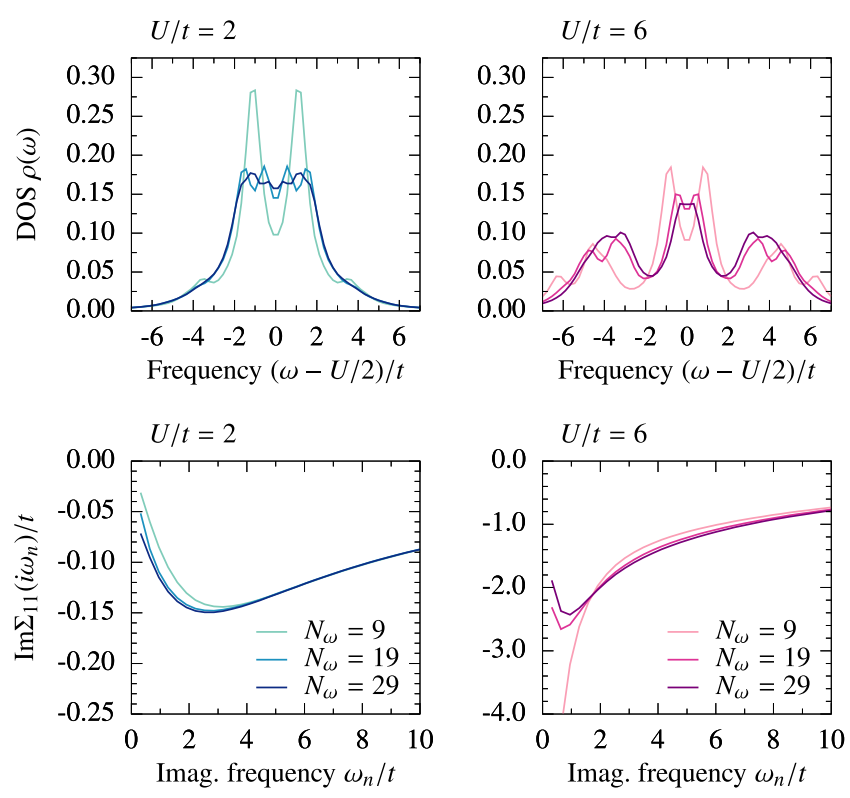

FIG. 2. Single-site DMFT results for the 1D Hubbard at half filling with weak interactions $(U / t=2$, left) and strong interactions $(U / t=6$, right), showing convergence with respect to the number of bath sites $N_{\omega}$. The chemical potential is fixed at $\mu=U / 2$ and the DOS is plotted with a broadening of $\eta / t=0.5$ for clarity.

we show that such artificial behavior can be removed, and the occupancy converges to the BA solution. This result may suggest that when discretizing the hybridization along the real axis, static quantities (e.g., occupancy) converge more slowly
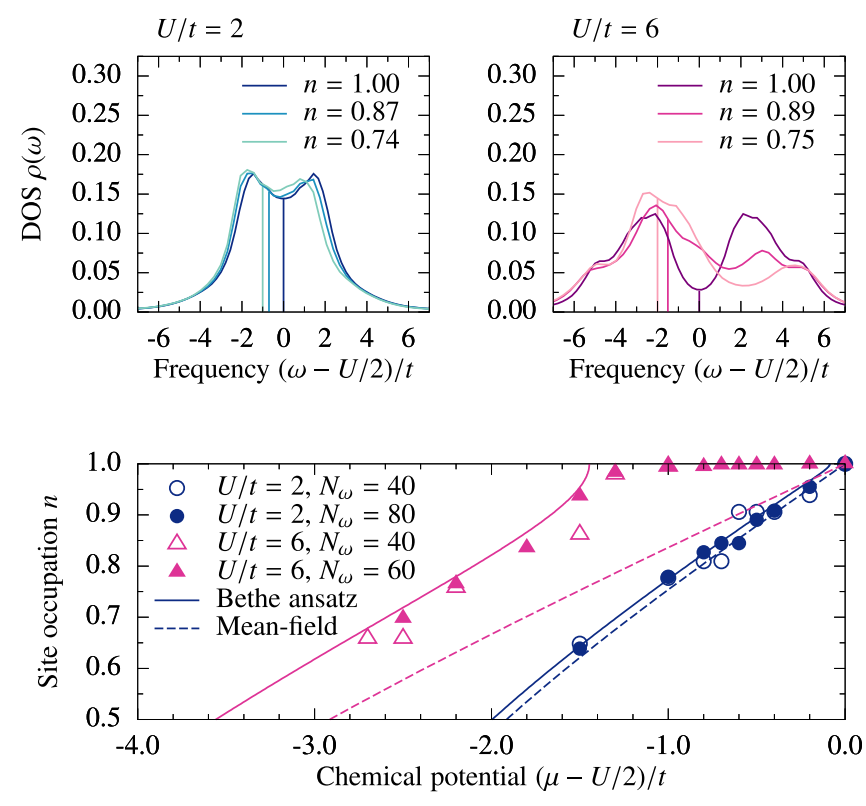

FIG. 3. Two-site CDMFT results as a function of doping. The DOS obtained using $N_{\omega}=30$ (60 bath sites in total) is shown in the top two panels, where the chemical potential is indicated by a vertical line. The site occupancy as a function of the chemical potential using $N_{\omega}=40$ (open symbols) and $N_{\omega}=60,80$ (filled symbols) is shown in the bottom panel, along with the numerically exact result from Bethe ansatz [61] and the mean-field result. 

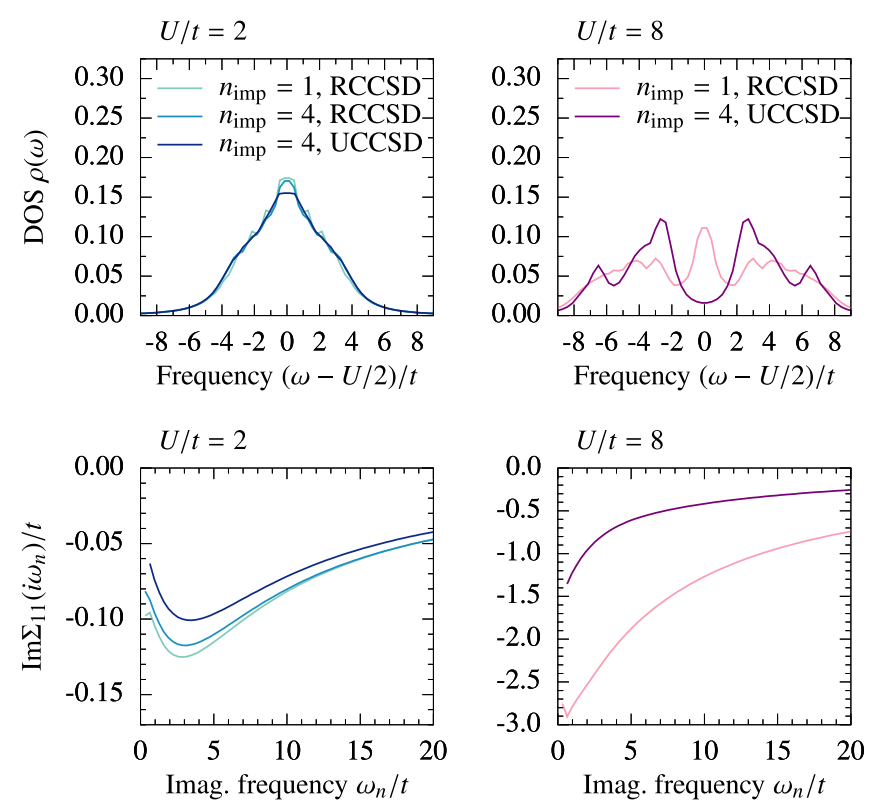

FIG. 4. Single-site DMFT and four-site $(2 \times 2)$ CDMFT results for the $2 \mathrm{D}$ Hubbard at half filling with weak interactions $(U / t=2$, left) and strong interactions $(U / t=8$, right). We used 30 bath sites for single-site DMFT and 120 bath sites for the four-site CDMFT. The chemical potential is fixed at $\mu=U / 2$ and the DOS is plotted with a broadening of $\eta / t=0.5$ for clarity.

with respect to the number of bath orbitals as compared to dynamic quantities (such as the spectral function), although a more careful comparison with imaginary-axis techniques is required.

\section{2D Hubbard model}

We next study the 2D Hubbard model at half filling. We perform calculations at $U / t=2$ and $U / t=8$. This is above and below the $2 \times 2$ CDMFT paramagnetic Mott transition around $U / t \approx 6[62,63]$. In Fig. 4, we show results for singlesite DMFT and $2 \times 2$ CDMFT. As before, we fix the number of bath sites to be about 30 per impurity site, such that our largest calculations have 120 particles in 120 orbitals.

At $U / t=2$ DMFT and CDMFT give very similar DOS and self-energies; i.e., the effect of the impurity size is small. Even if we allow for symmetry breaking in the $2 \times 2$ cluster, we find it to be very weak; thus (paramagnetic) RCCSD and (weakly antiferromagnetic) UCCSD give very similar results. In both cases, the gap is smaller than the bath discretization and thus indistinguishable from a metal. At $U / t=8$, paramagnetic single-site DMFT with RCCSD or UCCSD gives identical results, showing a Kondo-like feature centered between the lower and upper Hubbard bands. Paramagnetic $2 \times 2$ CDMFT with RCCSD fails to converge in the DMFT loop. The first few iterations of the DMFT cycle are physically reasonable and show the opening of a paramagnetic Mott gap. However, eventually an impurity problem is constructed for which the iterative solution of the ground-state RCCSD equations fails to converge, because the associated CC amplitudes become large. If we instead carry out an antiferromagnetic CDMFT calculation allowing for symmetry breaking in the
UCCSD solver, the DMFT cycle converges smoothly. As expected, the antiferromagnetic DOS exhibits a gap, with a qualitatively reasonable size of about $6 t$.

\section{CONCLUSIONS}

We have demonstrated the promise of coupled-cluster (CC) theory as an impurity solver in single-site dynamical meanfield theory and multisite cluster dynamical mean-field theory. In particular, the polynomial cost of truncated CC allows the use of many bath sites per impurity site, which enables a faithful discretization of the hybridization directly on the real frequency axis. In our studies of the Hubbard model, we find that the Green's functions and self-energies are well converged using approximately 30 bath sites per impurity site.

Despite the strongly correlated nature of the lattice problem, the low-order CCSD truncation provides very accurate results for spectral functions, self-energies, and occupation numbers as an impurity solver in DMFT, when there is a modest number of impurities. This is consistent with the impurity problem being less strongly correlated than the lattice problem, even for a dense discretization of the hybridization. Consequently, we expect that CC impurity solvers will find most use with clusters of a moderate size in real space. The low cost of the $\mathrm{CC}$ impurity solvers also makes them very promising for applications to real materials with many electrons and many orbitals per impurity site in $a b$ initio quantum chemical formulations of DMFT [13]. In particular, the embedding approach to $\mathrm{CC}$ response functions in solids is a promising alternative to full periodic $\mathrm{CC}$ calculations $[64,65]$, and work along these lines is currently in progress.

Note added. Recently Shee and Zgid have presented related work [66] that also explores the use of CC impurity solvers in Green's function embedding methods.

\section{ACKNOWLEDGMENTS}

T.Z. and G.K.C. were supported by the US Department of Energy, Office of Science, via Grant No. SC19390. G.K.C. is also supported by the Simons Foundation, via the ManyElectron Collaboration, and via the Simons Investigator Program. The Flatiron Institute is a division of the Simons Foundation.

\section{APPENDIX: CAUSALITY IN COUPLED-CLUSTER THEORY}

As discussed in Sec. II B, EOM-CCSD is not a conserving approximation and thus the resulting Green's functions and self-energies need not be causal. The lack of strict causality is not seen in any of the results we showed above. However, it is possible to observe small violations of causality if we carry out calculations with a very small broadening (e.g., $\eta=$ $0.001 t$ ). An example of noncausal behavior that can occur in this setting is shown in Fig. 5. As defined in Eq. (10), $\boldsymbol{\Sigma}_{\text {imp }}(\omega)$ should always have a negative imaginary part if it is computed from a causal impurity Green's function [30]. However, we see that the CCSD impurity self-energy develops a positive imaginary part exactly around the bath frequencies. One way to understand this is that truncated CC theory does not include 


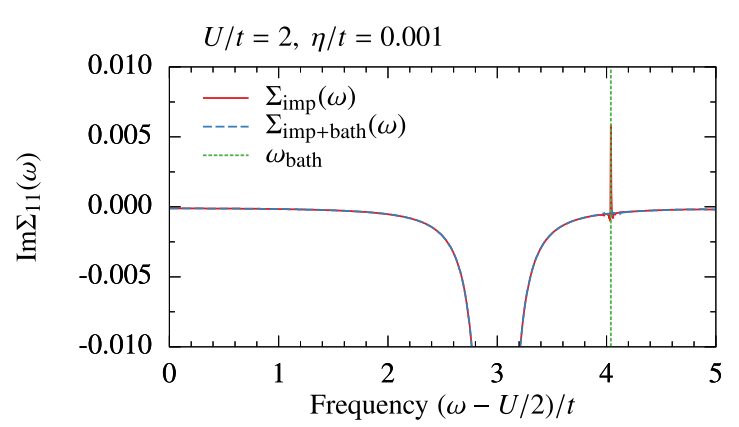

FIG. 5. Impurity self-energy on the real frequency axis from a two-site CDMFT calculation for the 1D Hubbard at half filling with $U / t=2$. RCCSD is used as the impurity solver and two bath sites are coupled to each impurity site. A small broadening of $\eta=0.001 t$ is used to show the noncausal behavior.

all possible diagrams involving interactions and hopping to the bath (i.e., the hybridization). Thus the hybridization contribution to $\mathbf{G}_{\text {imp }}^{-1}$ in Eq. (10) and $\boldsymbol{\Delta}(\omega)$ do not precisely cancel to give a causal self-energy.
This noncausality only appears when the broadening is very small and does not affect the results we presented. Nevertheless, we observe that there is a simple procedure that removes this issue in practice. Instead of computing $\boldsymbol{\Sigma}_{\text {imp }}(\omega)$ in Eq. (10), one can first compute the self-energy for the whole impurity plus bath system,

$$
\boldsymbol{\Sigma}_{\text {imp+bath }}(\omega)=\mathbf{G}_{0, \text { imp+bath }}^{-1}(\omega)-\mathbf{G}_{\text {imp+bath }}^{-1}(\omega),
$$

where $\mathbf{G}_{0, \text { imp+bath }}^{-1}(\omega)$ is the noninteracting Green's function of the impurity plus bath system. The impurity self-energy is then defined as the impurity block of $\boldsymbol{\Sigma}_{\text {imp+bath }}(\omega)$. [In an exact solver, this is the only nonzero part of $\boldsymbol{\Sigma}_{\text {imp+bath }}(\omega)$.] As shown in Fig. 5, the noncausal behavior disappears if the impurity self-energy is defined in this way, at the cost of more computation. While we do not claim that this procedure makes the self-energy strictly causal under all circumstances, we find that it removes even the small degree of noncausal behavior observed at very small broadenings in this work.
[1] A. Georges and G. Kotliar, Phys. Rev. B 45, 6479 (1992).

[2] A. Georges, G. Kotliar, W. Krauth, and M. Rozenberg, Rev. Mod. Phys. 68, 13 (1996).

[3] A. I. Lichtenstein and M. I. Katsnelson, Phys. Rev. B 62, R9283(R) (2000).

[4] G. Kotliar, S. Y. Savrasov, G. Pálsson, and G. Biroli, Phys. Rev. Lett. 87, 186401 (2001).

[5] M. H. Hettler, A. N. Tahvildar-Zadeh, M. Jarrell, T. Pruschke, and H. R. Krishnamurthy, Phys. Rev. B 58, R7475(R) (1998).

[6] M. H. Hettler, M. Mukherjee, M. Jarrell, and H. R. Krishnamurthy, Phys. Rev. B 61, 12739 (2000).

[7] E. Kozik, K. Van Houcke, E. Gull, L. Pollet, N. Prokof'ev, B. Svistunov, and M. Troyer, Europhysics Lett. 90, 10004 (2010).

[8] P. Werner, A. Comanac, L. de' Medici, M. Troyer, and A. J. Millis, Phys. Rev. Lett. 97, 076405 (2006).

[9] E. Gull, P. Werner, O. Parcollet, and M. Troyer, Europhysics Lett. 82, 57003 (2008).

[10] E. Gull, A. J. Millis, A. I. Lichtenstein, A. N. Rubtsov, M. Troyer, and P. Werner, Rev. Mod. Phys. 83, 349 (2011).

[11] M. Caffarel and W. Krauth, Phys. Rev. Lett. 72, 1545 (1994).

[12] M. Capone, L. de' Medici, and A. Georges, Phys. Rev. B 76, 245116 (2007).

[13] D. Zgid and G. K.-L. Chan, J. Chem. Phys. 134, 094115 (2011).

[14] K. G. Wilson, Rev. Mod. Phys. 47, 773 (1975).

[15] S. R. White, Phys. Rev. Lett. 69, 2863 (1992).

[16] R. Bulla, T. A. Costi, and T. Pruschke, Rev. Mod. Phys. 80, 395 (2008).

[17] M. C. Gutzwiller, Phys. Rev. Lett. 10, 159 (1963).

[18] J. Č́ízek, J. Chem. Phys. 45, 4256 (1966).

[19] S. Bravyi and D. Gosset, Commun. Math. Phys. 356, 451 (2017).

[20] D. Zgid, E. Gull, and G. K.-L. Chan, Phys. Rev. B 86, 165128 (2012).

[21] C. Lin and A. A. Demkov, Phys. Rev. B 88, 035123 (2013).
[22] Y. Lu, M. Höppner, O. Gunnarsson, and M. W. Haverkort, Phys. Rev. B 90, 085102 (2014).

[23] A. Go and A. J. Millis, Phys. Rev. B 96, 085139 (2017).

[24] A. F. White and G. K.-L. Chan, J. Chem. Theory Comput. 14, 5690 (2018).

[25] R. J. Bartlett, Annu. Rev. Phys. Chem. 32, 359 (1981).

[26] R. J. Bartlett and M. Musiał, Rev. Mod. Phys. 79, 291 (2007).

[27] T. A. Maier, M. Jarrell, T. Pruschke, and M. H. Hettler, Rev. Mod. Phys. 77, 1027 (2005).

[28] G. Kotliar, S. Y. Savrasov, K. Haule, V. S. Oudovenko, O. Parcollet, and C. A. Marianetti, Rev. Mod. Phys. 78, 865 (2006).

[29] K. Held, Adv. Phys. 56, 829 (2007).

[30] G. Biroli, O. Parcollet, and G. Kotliar, Phys. Rev. B 69, 205108 (2004).

[31] M. Capone, M. Civelli, S. S. Kancharla, C. Castellani, and G. Kotliar, Phys. Rev. B 69, 195105 (2004).

[32] I. de Vega, U. Schollwöck, and F. A. Wolf, Phys. Rev. B 92, 155126 (2015).

[33] A. Liebsch and H. Ishida, J. Phys.: Condens. Matter 24, 053201 (2012).

[34] E. Koch, G. Sangiovanni, and O. Gunnarsson, Phys. Rev. B 78, 115102 (2008).

[35] F. A. Wolf, A. Go, I. P. McCulloch, A. J. Millis, and U. Schollwöck, Phys. Rev. X 5, 041032 (2015).

[36] R. Peters, Phys. Rev. B 84, 075139 (2011).

[37] M. Ganahl, P. Thunström, F. Verstraete, K. Held, and H. G. Evertz, Phys. Rev. B 90, 045144 (2014).

[38] M. Ganahl, M. Aichhorn, H. G. Evertz, P. Thunström, K. Held, and F. Verstraete, Phys. Rev. B 92, 155132 (2015).

[39] I. Shavitt and R. J. Bartlett, Many-Body Methods in Chemistry and Physics (Cambridge University Press, Cambridge, 2009).

[40] G. E. Scuseria, C. L. Janssen, and H. F. Schaefer, J. Chem. Phys. 89, 7382 (1988). 
[41] M. Nooijen and J. G. Snijders, Int. J. Quantum Chem. 44, 55 (1992).

[42] M. Nooijen and J. G. Snijders, Int. J. Quantum Chem. 48, 15 (1993).

[43] J. McClain, J. Lischner, T. Watson, D. A. Matthews, E. Ronca, S. G. Louie, T. C. Berkelbach, and G. K.-L. Chan, Phys. Rev. B 93, 235139 (2016).

[44] K. Bhaskaran-Nair, K. Kowalski, and W. A. Shelton, J. Chem. Phys. 144, 144101 (2016).

[45] J. F. Stanton and R. J. Bartlett, J. Chem. Phys. 98, 7029 (1993).

[46] H. J. Monkhorst, Int. J. Quantum Chem. 12, 421 (2009).

[47] A. I. Krylov, Annu. Rev. Phys. Chem. 59, 433 (2008).

[48] V. M. Galitskii and A. B. Migdal, Sov. Phys. JETP 7, 96 (1958).

[49] M. F. Lange and T. C. Berkelbach, J. Chem. Theory Comput. 14, 4224 (2018).

[50] S. R. White and A. E. Feiguin, Phys. Rev. Lett. 93, 076401 (2004).

[51] E. Ronca, Z. Li, C. A. Jimenez-Hoyos, and G. K.-L. Chan, J. Chem. Theory Comput. 13, 5560 (2017).

[52] Y. Saad and M. H. Schultz, SIAM J. Sci. Stat. Comput. 7, 856 (1986).

[53] E. de Sturler, SIAM J. Numer. Anal. 36, 864 (1999).
[54] P. Pulay, Chem. Phys. Lett. 73, 393 (1980).

[55] Q. Sun, T. C. Berkelbach, N. S. Blunt, G. H. Booth, S. Guo, Z. Li, J. Liu, J. D. McClain, E. R. Sayfutyarova, S. Sharma, S. Wouters, and G. K.-L. Chan, Wiley Interdiscip. Rev. Comput. Mol. Sci. 8, e1340 (2018).

[56] J. Hubbard, Proc. R. Soc. London, Ser. A 276, 238 (1963).

[57] A. Liebsch, H. Ishida, and J. Merino, Phys. Rev. B 78, 165123 (2008).

[58] A. Liebsch and N.-H. Tong, Phys. Rev. B 80, 165126 (2009).

[59] M. J. Rozenberg, G. Kotliar, and H. Kajueter, Phys. Rev. B 54, 8452 (1996).

[60] C. J. Bolech, S. S. Kancharla, and G. Kotliar, Phys. Rev. B 67, 075110 (2003).

[61] E. H. Lieb and F. Y. Wu, Phys. Rev. Lett. 20, 1445 (1968).

[62] H. Park, K. Haule, and G. Kotliar, Phys. Rev. Lett. 101, 186403 (2008).

[63] G. Sordi, K. Haule, and A.-M. S. Tremblay, Phys. Rev. Lett. 104, 226402 (2010).

[64] J. McClain, Q. Sun, G. K.-L. Chan, and T. C. Berkelbach, J. Chem. Theory Comput. 13, 1209 (2017).

[65] T. Gruber, K. Liao, T. Tsatsoulis, F. Hummel, and A. Grüneis, Phys. Rev. X 8, 021043 (2018).

[66] A. Shee and D. Zgid, arXiv:1906.04079. 